\title{
Editorial
}

\section{Welcome to a New Journal-Journal of Gene Regulation}

\author{
Glen M. Borchert ${ }^{1,2}$ and Yaguang $\mathrm{Xi}^{3}$ \\ ${ }^{1}$ Department of Pharmacology, University of South Alabama, Mobile, AL 36688, USA \\ ${ }^{2}$ Department of Biology, University of South Alabama, Mobile, AL 36608, USA \\ ${ }^{3}$ Department of Genetics and Stanley S. Scott Cancer Center, Louisiana State University Health Sciences Center, New Orleans, LA 70112, USA \\ Address correspondence to Glen M. Borchert, borchert@southalabama.edu; Yaguang Xi, yxi@lsuhsc.edu
}

Received 5 May 2021; Accepted 5 May 2021

Copyright () 2021 Glen M. Borchert and Yaguang Xi. This is an open access article distributed under the terms of the Creative Commons Attribution License, which permits unrestricted use, distribution, and reproduction in any medium, provided the original work is properly cited.

Gene regulation refers to how a cell controls which genes, out of the many genes in its genome, are "turned on" (expressed) or "turned off" (repressed). Different cells in a multicellular organism may express very different sets of genes, even though they contain the same DNA. The full set of genes expressed in a cell includes the total complement of proteins and functional RNAs it produces. Gene expression can be controlled at various stages, from the accessibility of DNA to the production and processing of mRNAs to the translation and activation of proteins.

Over the last two decades, our understanding of the complexity of gene regulation has improved dramatically, largely due to the invention of high throughput RNAsequencing (RNA-seq). A host of recent RNA-seq studies, in species ranging from bacteria to humans, has revealed that the transcribed portions of most genomes are much more extensive than originally thought, and that most genes actually encode noncoding RNAs (ncRNAs), rather than proteins. Importantly, in addition to this raising numerous new questions and insights regarding the regulation of the expression of these novel ncRNAs themselves, it has become increasingly apparent that these ncRNAs are not merely nonfunctional "junk" transcripts, but that a significant number of ncRNAs serve as functional molecules involved in the regulation of transcriptional and post-transcriptional gene expression.

Sequence-specific transcription factors were traditionally considered the most important and defining components of gene regulation in both prokaryotic and eukaryotic cells. That said, we now know gene regulation is much more complex. For example, the state of chromatin structure at a specific region in eukaryotic DNA, along with the presence of specific transcription factors, coordinates to regulate gene expression. However, this complex interplay between transcriptional activators or repressors and accessibility of genomic regulatory sequences is still only a small part of the story. Epigenetic mechanisms, including DNA methylation and imprinting, noncoding RNAs, post-translational modifications, and other mechanisms, further enrich the cellular portfolio of gene expression controls.

However, while numerous journals routinely publish reports on the underlying mechanisms and medical relevance of gene regulation, no journal currently exists dedicated specifically to the complex and pivotal field of gene expression. As such, it is quite challenging to keep fully updated in the area with relevant articles spread widely across the primary literature. The Journal of Gene Regulation (JGR) aims therefore to serve as a gathering point for the publication of original research findings and comprehensive reviews that facilitate advances in our mechanistic understanding of genetic and epigenetic controls of gene expression in prokaryotes, eukaryotes, and viruses. All aspects of gene expression will be examined through publication of high-quality original papers, reviews, opinions, clinical case reports, debates, and invited editorials. As Editors-in-Chief of the Journal of Gene Regulation (JGR), we will be dedicated to disseminating peer-reviewed basic, experimental, and clinical research related to this keystone field, specifically including all aspects of

- Epigenetics, chromatin remodeling and genomic feature compositions (e.g., promoters, enhancers, insulators), and genomic modification (e.g., methylation)

- RNA polymerase and transcription factor binding and regulation

- RNA stability and RNA processing (e.g., splicing, capping, polyadenylation, and editing)

- Translational regulation and protein activation mechanisms

- Internal and external signal transduction

- Noncoding RNA regulators of gene expression

- New genome editing technologies 
- Gene therapy

- RNA drug development.

The goal of the Journal of Gene Regulation (JGR) is to improve the understanding of gene regulatory mechanisms. We sincerely welcome multidisciplinary submissions to be considered for publication in this new peer-reviewed journal on topics including molecular biology, microbiology, genetics, immunology, bioinformatics, oncology, pharmacology, and comparative studies. Studies that can develop the impact of gene regulation on the treatment of human diseases are also highly encouraged.

All articles published in the Journal of Gene Regulation (JGR) are open access and distributed under the Creative Commons Attribution License, which permits unrestricted use, distribution, and reproduction in any medium, provided the original work is properly cited. For more information regarding the Journal of Gene Regulation (JGR), including our distinguished Board of Associate Editors, we invite you to visit our website (http://www.bendola.com/journals/jgr/).

Glen M. Borchert and Yaguang Xi Editors-in-Chief 